old the employees perceive to be) shape the formation of age-based subgroups in organizations more strongly than the chronological age of employees does. Shared perceptions of an age discrimination climate are in turn expected to be positively related to the average level of reported psychological health (measured through burnout perceptions) in organizations, with stronger effects for older compared to younger employees, proposing a moderated-mediation model. Our study hypotheses are tested and supported in a sample of 96 German small and medium-sized companies with more than 16,000 employees.

\section{RETIREMENT AND COGNITIVE FUNCTIONING: A TRICKY ASSOCIATION}

\section{Grotz, S. Adam, University of Liège, Liège, Belgium}

While different types of activities are associated with cognition, regardless of past activities, people aged over 60 can still have some control over their cognition through the maintenance of activities (e.g., a 60-year-old individual can delay his cognitive aging by 1.75 year if he engages in charity/voluntary work, or by 1.38 year if he continues employment). Later retirement ages could also delay the onset of neurodegenerative diseases. However, the transition from work to retirement is not simply an objective life event but is also a major life process that is marked by numerous changes (e.g., social networks). Individuals experience the retirement transition and adapt to it in different ways. In this respect, we examined the influence of psychological transition and retirement-related adjustment processes on cognition. Results showed that positive consideration towards work and development of new activities after retirement are determinants of good cognitive functioning at retirement.

\section{SPILLOVER AND 10-YEAR CHANGE IN HEALTH: THE ROLE OF PERSONALITY}

D. Segel-Karpas ${ }^{1}$, S. Agrigoroaei², 1. Gerontology, University of Haifa, Haifa, Israel, 2. Université catholique de Louvain, Louvain-la-Neuve, Belgium

Balancing between the work and family demands is psychologically demanding, and the transfer of negative affect and worries from work to home was found to harm workers' health and well-being. We used data from two waves of the Midlife in the US national longitudinal study $(\mathrm{N}=3411)$ to examine whether work-to-family spillover has negative consequences in terms of 10-year change in physical health, and whether this association is moderated by the Big 5 personality traits. As expected, spillover was significantly associated with residual change in health, operationalized as the number of chronic conditions. Moreover, the negative consequences on health were higher for those with higher levels of Conscientiousness and Agreeableness. In contrast, higher levels of Openness to Experience reduce the effects of spillover on health. The results are discussed with regard to the individual differences in terms of abilities to cope with work to family spillover.

\section{RETIREMENT AND TRAJECTORIES OF CHANGE IN SPEED OF INFORMATION PROCESSING}

R. Andel ${ }^{1}$, P. Butterworth ${ }^{2}$, N. Cherbuin ${ }^{3}$, K. Anstey ${ }^{3}$, 1. School of Aging Studies, University of South Florida, Tampa, Florida, 2. University of Melbourne, Melbourne, Victoria, Australia, 3. Australian National University, Canberra, Australian Capital Territory, Australia

IAGG 2017 World Congress
Retirement signifies a key transitional period. We examined retirement and change in processing speed, a prime indicator of cognitive aging, in 1405 Australians from the community-based Personality and Total Health (PATH) Project. Baseline age was $63(\mathrm{SD}=2)$ years, $36 \%$ were women, average education was 14 years. Symbol Digit Modalities Test was administered four times over 12 years to measure speed. Retirement was a time-varying variable. In random effects models adjusted for baseline age, retirement age, sex, education, self-rated health, and work complexity, retiring was associated with accelerated decline in speed (Estimate $=-0.15$, $\mathrm{p}<.001)$. This effect was about 7 times greater than the corresponding effect of years of age (Estimate=-0.02). Retiring due to illness, being laid off, or the desire to pursue other activities did not explain the results. Retirement age did not modify the effect. Transition into retirement may be an important period with respect to the trajectory of cognitive aging.

\section{SESSION 4815 (SYMPOSIUM)}

\section{PRESIDENTIAL SYMPOSIUM: NEW EVIDENCE ON COGNITIVE AND BRAIN AGEING FROM THE DISCONNECTED MIND PROJECT}

Chair: T. Wright, Age UK, London, United Kingdom

Discussant: J. Goodwin, Age UK, London, United Kingdom

Building a detailed picture of cognitive and brain ageing is imperative for predicting and ameliorating functional decline in later life. This symposium showcases recent work from the Disconnected Mind project. It centres around the Lothian Birth Cohort 1936, a group of Scottish older people who sat a cognitive test at age 11 in 1947 . They were followed up on three occasions, aged 70,73 , and 76 years, the latter two waves including an MRI scan. First, Stuart Ritchie asks whether there is structure in declines across many different cognitive abilities, assessing which lifestyle, genetic, socioeconomic, and health measures are the best predictors of these declines. Second, Simon Cox asks a similar question regarding brain measurements: which variables make predictions of later-life changes in important brain parameters, and how do they interact with one another? Third, Benjamin Aribisala zooms in more deeply on the brain, reporting new analyses of the hippocampus. This memory-linked structure can be imaged in multiple ways, some of which provide better information regarding cognitive decline than others. Finally, Sherif Karama discusses links between cognitive abilities and the thickness of the brain's cortex in later life. He makes a more general point about aging research, one on which the Disconnected Mind project can make a unique contribution: researchers must take into account pre-existing cognitive ability before drawing conclusions regarding later-life cognitive-brain associations. Overall, the seminar gives an overview of recent developments in understanding the process, biology, and determinants of cognitive aging - and their implications for policy and practice.

\section{STRUCTURE AND PREDICTORS OF COGNITIVE CHANGE IN THE EIGHTH DECADE OF LIFE}

S. Ritchie ${ }^{1}$, E. Tucker-Drob ${ }^{2}$, I.J. Deary ${ }^{1}, 1$. University of Edinburgh, Edinburgh, United Kingdom, 2. University of Texas, Austin, Texas 